Research Article

\title{
Dual-Band $2 \times 2$ MIMO Antenna with Compact Size and High Isolation Based on Half-Mode SIW
}

\author{
Abubaker Ahmed Elobied $\mathbb{D},{ }^{1}$ Xue-Xia Yang $\mathbb{D}^{2},{ }^{2}$ Ningjie Xie $\mathbb{D}^{1},{ }^{1}$ and Steven Gao $\mathbb{D}^{3}$ \\ ${ }^{1}$ School of Communication and Information Engineering, Shanghai University, Shanghai 200444, China \\ ${ }^{2}$ Key Laboratory of Specialty Fiber Optics and Optical Access Networks, \\ The Shanghai Institute for Advanced Communication and Data Science, \\ Joint International Research Laboratory of Specialty Fiber Optics and Advanced Communication, Shanghai University, \\ Shanghai 200444, China \\ ${ }^{3}$ School of Engineering and Digital Arts, University of Kent, Canterbury CT2 7NZ, UK
}

Correspondence should be addressed to Xue-Xia Yang; yang.xx@shu.edu.cn

Received 3 August 2020; Revised 11 October 2020; Accepted 13 November 2020; Published 27 November 2020

Academic Editor: Mauro Parise

Copyright (C) 2020 Abubaker Ahmed Elobied et al. This is an open access article distributed under the Creative Commons Attribution License, which permits unrestricted use, distribution, and reproduction in any medium, provided the original work is properly cited.

\begin{abstract}
This paper presents a close-spaced dual-band $2 \times 2$ multiple-input multiple-output (MIMO) antenna with high isolation based on half-mode substrate integrated waveguide (HMSIW). The dual-band operation of the antenna element is achieved by loading a rectangular patch outside the radiating aperture of an HMSIW cavity. The HMSIW cavity is excited by a coaxial probe, whereas the rectangular patch is energized through proximity coupling by the radiating aperture of HMSIW. The antenna elements can be closely placed using the rotation and orthogonal arrangement for a $2 \times 2$ array. Small neutralization lines at the center of the MIMO antenna can increase the isolation among its elements by around $10 \mathrm{~dB}$ in the lower band and $5 \mathrm{~dB}$ in the higher band. A prototype of the MIMO antenna is fabricated and its performance is measured. The measured results show that the resonant frequencies are centered at 4.43 and $5.39 \mathrm{GHz}$ with bandwidths of 110 and $80 \mathrm{MHz}$ and peak gains of 6 and $6.4 \mathrm{dBi}$, respectively. The minimum isolation in both bands is greater than $35 \mathrm{~dB}$. The envelope correlation coefficient is lower than 0.005 within two operating bands.
\end{abstract}

\section{Introduction}

Antenna systems that support multiple wireless standards and have compact size are highly desirable for future portable devices. Multiple-input multiple-output (MIMO) antenna technology has become essential for future portable devices owing to its ability to increase data throughput without increasing the power and bandwidth [1]. Therefore, developing compact multiband antennas to support multifunctional MIMO systems is necessary. High isolation among the elements of a compact dual-band MIMO antenna is important for the performance of a communication system.

Some dual-band MIMO antennas obtained using different decoupling techniques have been introduced in the literature; these are generally summarized into two types. The first type involves decoupling antenna elements without inserting any decoupling structures; here, the decoupling could be realized using spatial [2], pattern [3], and polarization diversities [4]. Spatial diversity can be achieved by arranging two or more antenna elements such that there is a wide space between them; therefore, such MIMO antennas have a large size. Pattern diversity can be realized by discriminating antenna elements over an angled space, whereas polarization diversity can be achieved by orthogonally arranging the antenna elements. Pattern and polarization diversities are preferred because the elements can be arranged close to each other. However, the coupling is difficult to control when more than two closely spaced antennas are employed. 
The second type of decoupling for MIMO antenna involves inserting a decoupling structure between the antenna elements to block or suppress the coupling, which includes defected ground structure [5], complementary split-ring resonator [6], electromagnetic bandgap [7], neutralization line [8], T-shaped stub [9], and ring strip [10]. However, these structures increase the space between antenna elements, which results in larger antenna sizes. High isolation can be achieved by combining the two methods above [11-15].

In this paper, a dual-band antenna element is proposed by loading a rectangular patch outside the aperture of a halfmode substrate integrated waveguide (HMSIW) cavity. By combining the spatial and polarization diversities and using small neutralization lines (SNLs), a close-spaced $2 \times 2$ MIMO antenna with high isolation and dual-band operation is suggested. To validate the proposed antenna, a prototype is fabricated and its performance is measured. This paper is arranged as follows. In Section 2, the design procedure of the proposed dual-band antenna element is discussed. In Section 3, four-element MIMO antennas are introduced and their corresponding decoupling mechanisms are explained. The fabrication and measurement results are presented in Section 4 . The conclusion of the proposed work is presented in Section 5.

\section{Dual-Band Antenna Element}

A dual-band antenna element obtained by loading a rectangular patch outside an HMSIW cavity is proposed, as shown in Figure 1. The antenna element has only one substrate layer, and the bottom side of the substrate is a metallic ground plane. The HMSIW antenna is fed by a coaxial probe, whereas the patch antenna is excited through proximity coupling by the radiating aperture of the HMSIW antenna. The HMSIW antenna generates the lower band, whereas the loading patch generates the upper band.

The geometrical size of the proposed dual-band antenna element is shown in Figure 2. The size of the HMSIW cavity is $W \times W$ and the dimensions of the patch are $L_{p} \times W_{p}$. The coupling gap is designated as $G$. To be equivalent to a conventional metallic cavity, the diameter $d$ and pitch $s$ of the HMSIW cavity via holes must meet the conditions of $d$ / $s \geq 0.5$ and $d / \lambda_{0} \leq 0.1[16]$.

The antenna element was designed on a single-layer substrate of Rogers Duroid 5880 with a relative dielectric constant of 2.2, tangent loss of 0.0009 , and thickness of $1.57 \mathrm{~mm}$. A high-frequency structure simulator was used to analyze and simulate the antenna. The optimum parameters of the proposed antenna element are $W=40 \mathrm{~mm}$, $L_{p}=18 \mathrm{~mm}, W_{p}=20 \mathrm{~mm}$, and $G=0.4 \mathrm{~mm}$. The simulated reflection coefficient of the proposed dual-band antenna is depicted in Figure 3. The two simulated resonances appear at 4.46 and $5.38 \mathrm{GHz}$.

Figure 4 depicts the simulated electric field distributions at $f_{L}=4.46 \mathrm{GHz}$ and $f_{H}=5.38 \mathrm{GHz}$. Figure $4(\mathrm{a})$ illustrates that the power is concentrated along the edge of the aperture of the HMSIW cavity, which implies that the resonant mode at $f_{L}=4.46 \mathrm{GHz}$ is generated by the HMSIW cavity. In contrast, the electric field is mainly concentrated along the two edges of the patch at $f_{H}=5.38 \mathrm{GHz}$, as observed in Figure 4(b).

\section{Dual-Band $2 \times 2$ MIMO Antenna}

3.1. Four-Element Antenna Configuration. An initial configuration of the array is proposed by replicating and rotating the antenna element described in Section 2. As shown in Figure 5, the element pairs $(1,4)$ and $(2,3)$ provide spatial diversity, whereas the orthogonal arrangement of the pairs $(1,2)$ and $(1,3)$ results in polarization diversity. Good isolation was achieved by exploiting the spatial and polarization diversities. The ground planes of the antenna elements were separated by distance $g=2 \mathrm{~mm}$ to further improve the isolation. The dimensions of the MIMO antenna are $82 \times 82 \mathrm{~mm}$.

The simulated S-parameters of the four-element MIMO antenna are depicted in Figure 6. Owing to the symmetrical arrangement, $\quad\left|S_{12}\right|=\left|S_{42}\right|=\left|S_{34}\right|=\left|S_{13}\right|$ and $\left|S_{14}\right|=\left|S_{23}\right|$; therefore, only $\left|S_{11}\right|,\left|S_{12}\right|,\left|S_{13}\right|$, and $\left|S_{14}\right|$ are presented here. $S_{11}$ demonstrated that the antenna resonated at 4.46 and $5.38 \mathrm{GHz}$, which are the same resonance frequencies as those of the element in Figure 3. The minimum isolation levels were better than $25 \mathrm{~dB}$ in both bands.

3.2. Isolation Enhancement of the Four-Element Array. As shown in Figure 7, four SNLs were inserted between the radiators of the MIMO antenna to further reduce the coupling current without increasing the size of the original MIMO antenna. The SNLs created additional current paths to transfer some of the current from one element and feed it back to another element with a suitable magnitude and an opposite phase, which eliminates the coupling. Each SNL was placed away from the open end of the antenna element in a low impedance area, where the current has the highest intensity. The decoupling current can be controlled by adjusting the location of the SNLs. Figure 8 shows the effects of location $d_{n l}$ on the isolation. Isolation higher than $35 \mathrm{~dB}$ in both bands was achieved when the location $d_{n l}$ was $5 \mathrm{~mm}$.

To further understand the contribution of the SNLs to isolation enhancement, the simulated current distributions with and without SNLs at 4.46 and $5.38 \mathrm{GHz}$ are illustrated in Figure 9. The current intensity in the other elements $(2,3$, and 4) is reduced when element 1 is excited and the SNLs are introduced. Figure 9 shows that the SNLs are more effective in the lower band than in the upper band.

Figure 10 presents the simulated S-parameters of the $2 \times 2$ MIMO antenna with the SNLs structure. The two simulated resonances appear at 4.46 and $5.38 \mathrm{GHz}$ and the isolation in both bands is higher than $35 \mathrm{~dB}$. This means the isolation in both bands increased by around $10 \mathrm{~dB}$ in comparison with the MIMO antenna without SNLs.

\section{Fabrication and Measurement}

As shown in Figure 11, a prototype of the proposed MIMO antenna was fabricated to verify its effectiveness. 


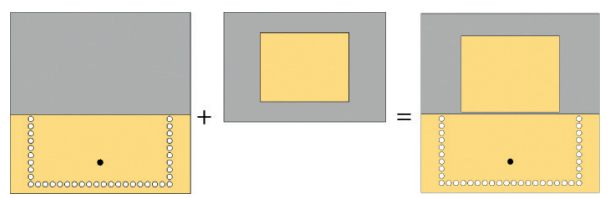

FIgURE 1: Construction of the proposed antenna element.

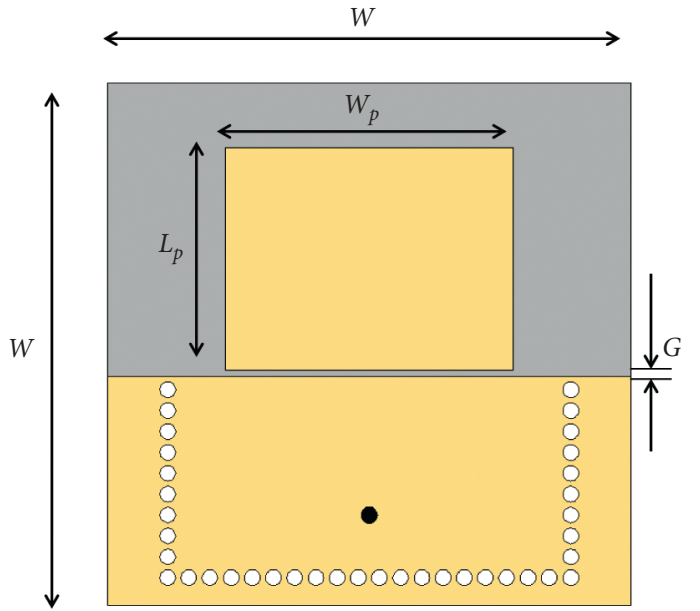

(a)

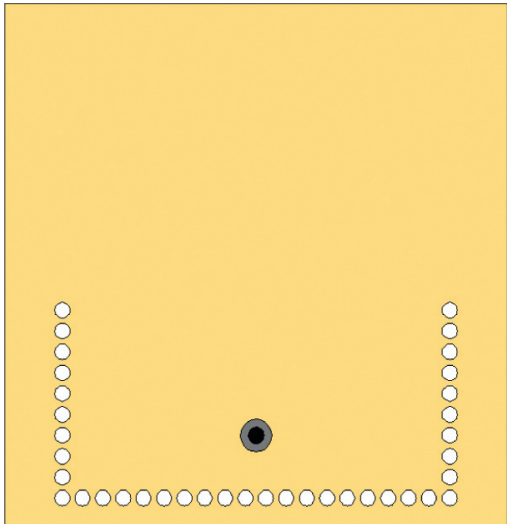

(b)

Figure 2: Geometry of the proposed dual-band antenna element. (a) Top view and (b) bottom view.

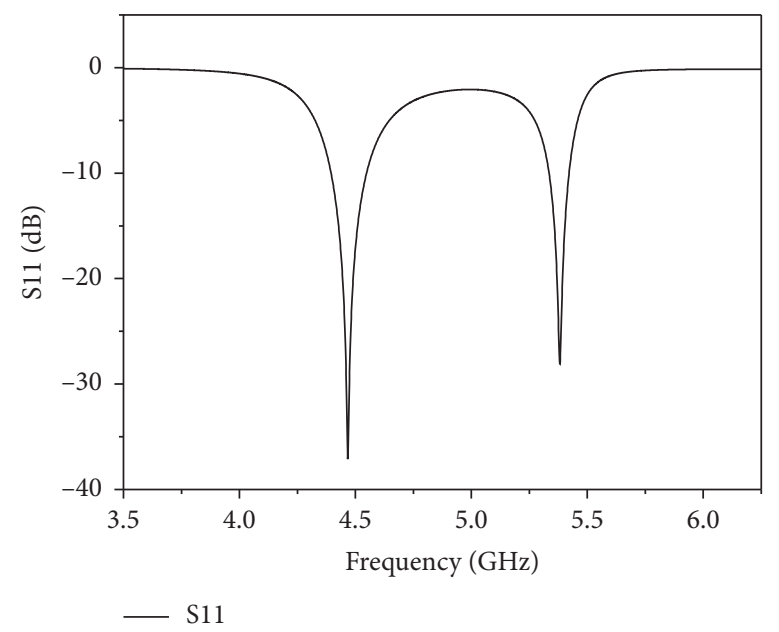

FIgURE 3: Simulated reflection coefficient of the proposed dual-band antenna.

4.1. S-Parameters. An Agilent N5227A vector network analyzer was used to measure the S-parameters. As shown in Figure 12, the measured $S_{11}$ resonated at 4.43 and $5.39 \mathrm{GHz}$. The measured minimum isolation levels in both bands are higher than $35 \mathrm{~dB}$. These results are in good agreement with the simulation results.

4.2. Radiation Patterns. The radiation patterns of the fabricated antenna were measured in an anechoic chamber and compared with the simulation results. Owing to the symmetry of the antenna structure, only the radiation patterns of element 1 are shown. As shown in Figure 13, the simulated and measured radiation patterns at 4.46 and $5.38 \mathrm{GHz}$ are in good agreement. The measured gains are 6 and $6.4 \mathrm{dBi}$, respectively, and the cross-polarization levels are lower than $-14 \mathrm{~dB}$ in both bands. These results are in good agreement with the simulation results.

4.3. Diversity Performance. The diversity performance of the proposed MIMO antenna is described in terms of envelope 


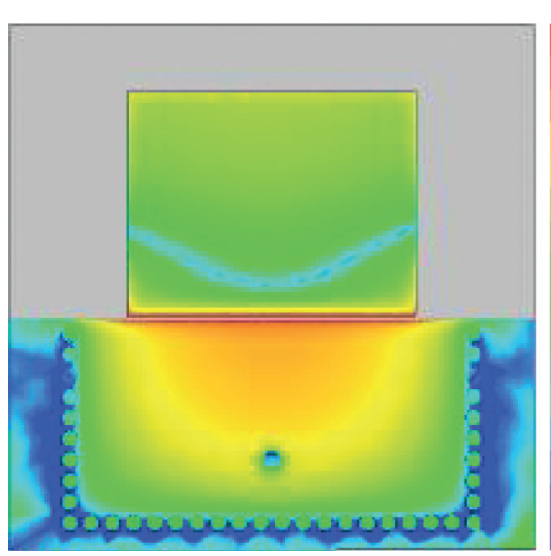

(a)
E Field [V_per_m]

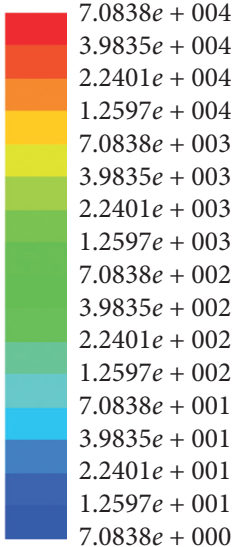

$7.0838 e+000$

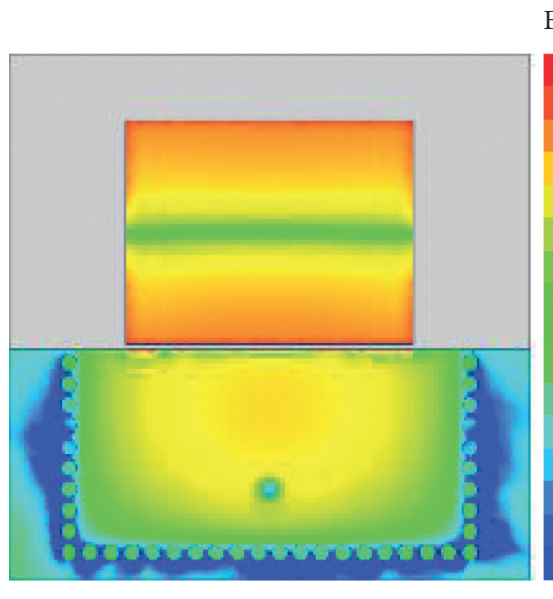

(b)
E Field [V_per_m]

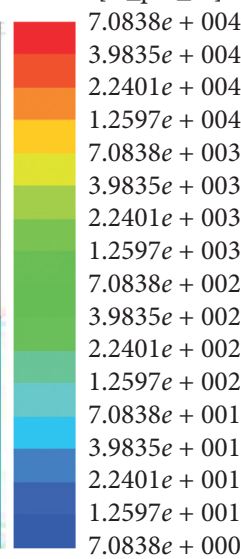

Figure 4: Simulated electric field distribution on the dual-band antenna at the two resonant frequencies: (a) $f_{L}=4.46 \mathrm{GHz}$ and (b) $f_{H}=5.38 \mathrm{GHz}$.

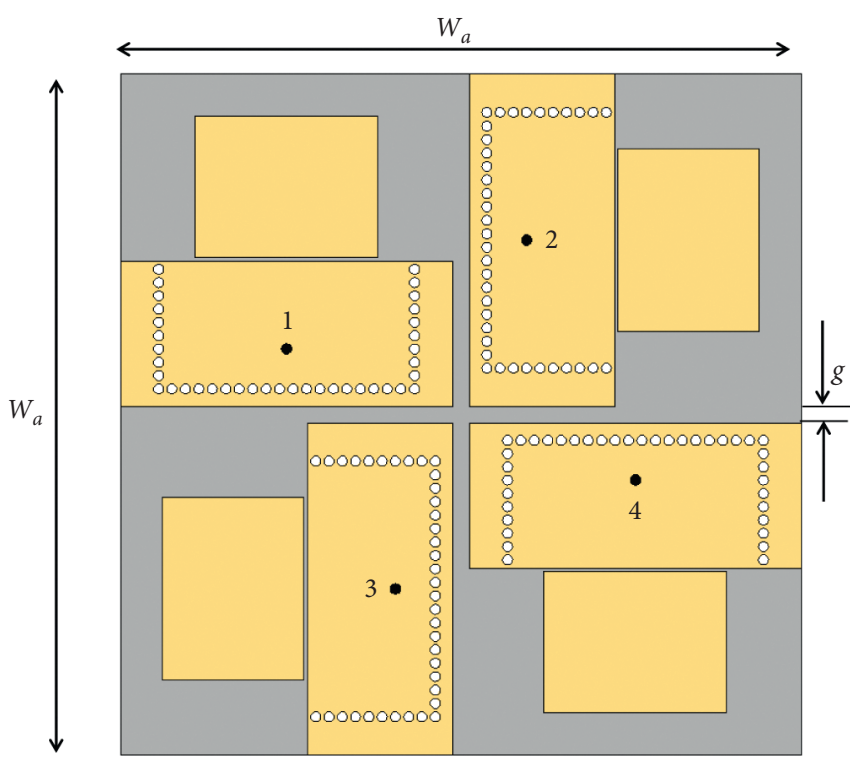

(a)

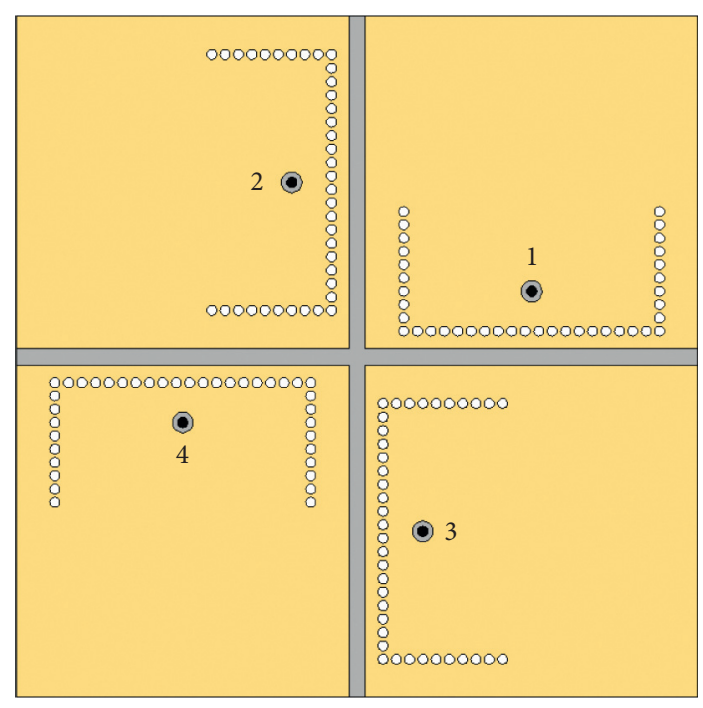

(b)

FIgURE 5: Geometry of the MIMO antenna. (a) Top view and (b) bottom view.

correlation coefficient (ECC), diversity gain (DG), total active reflection coefficient (TARC), mean effective gain (MEG), and channel capacity loss (CCL).

ECC is considered a paramount factor in the analysis of MIMO antennas. For better performance of the MIMO antenna, ECC $<0.5$ across the operating frequency is essential. The ECC can be computed by using either the S-parameters [15] or three-dimensional far fields [12]:

$$
\begin{aligned}
& \mathrm{ECC}=\rho_{i j}=\frac{\left|S_{i i}^{*} S_{i j}+S_{i j}^{*} S_{j j}\right|^{2}}{\left(1-\left|S_{i i}\right|^{2}-\left|S_{j i}\right|^{2}\right)\left(1-\left|S_{j j}\right|^{2}-\left|S_{i j}\right|^{2}\right)}, \\
& \mathrm{ECC}=\rho_{i j}=\frac{\left|\iint_{4 \pi}\left[F_{i}(\theta, \varphi) \cdot F_{j}^{*}(\theta, \varphi)\right] \mathrm{d} \Omega\right|^{2}}{\iint_{4 \pi}\left|F_{i}(\theta, \varphi)\right|^{2} \mathrm{~d} \Omega \iint_{4 \pi}\left|F_{j}(\theta, \varphi)\right|^{2} \mathrm{~d} \Omega,}
\end{aligned}
$$




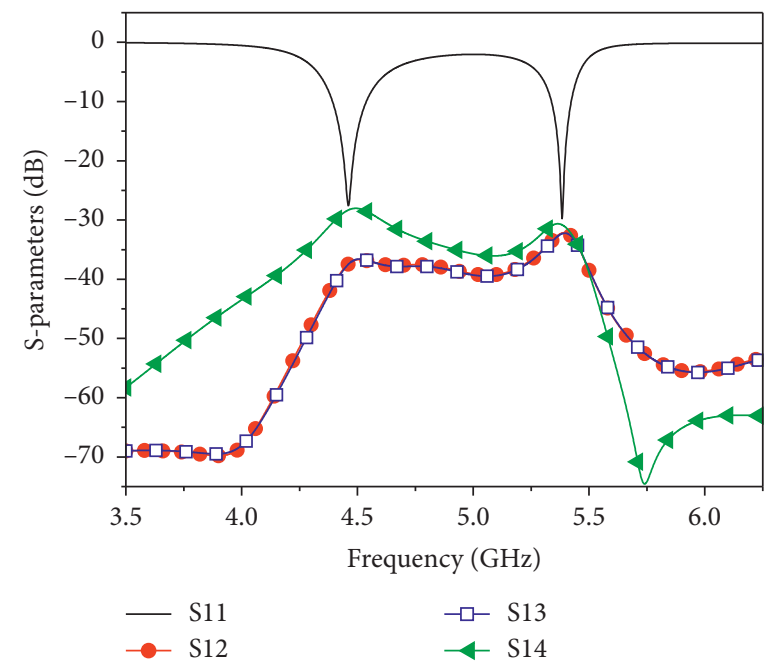

FIgURE 6: Simulated S-parameters of the four-element dual-band MIMO antenna.

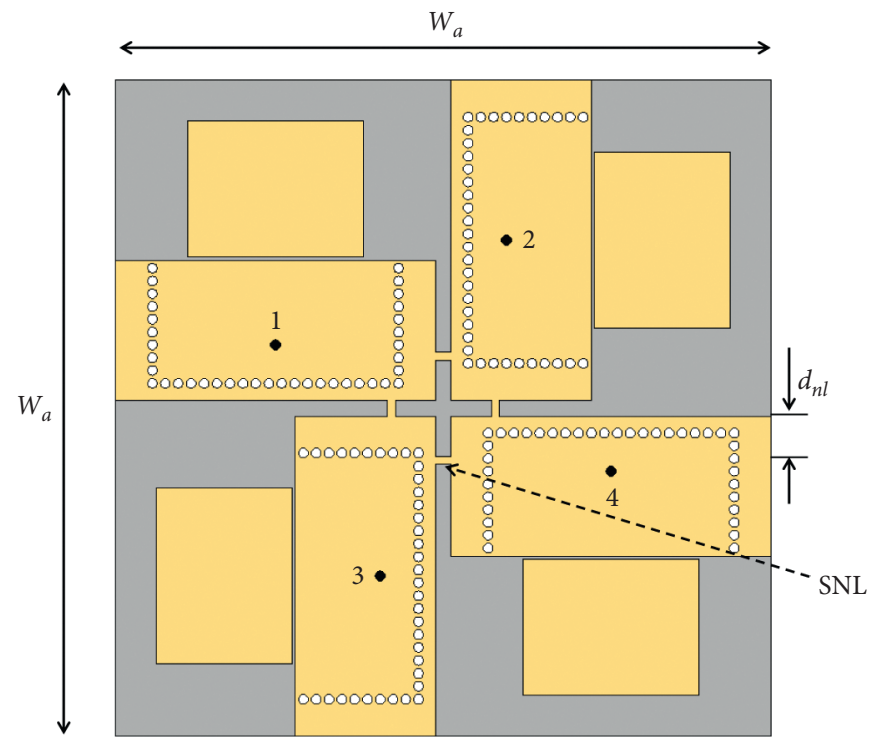

(a)

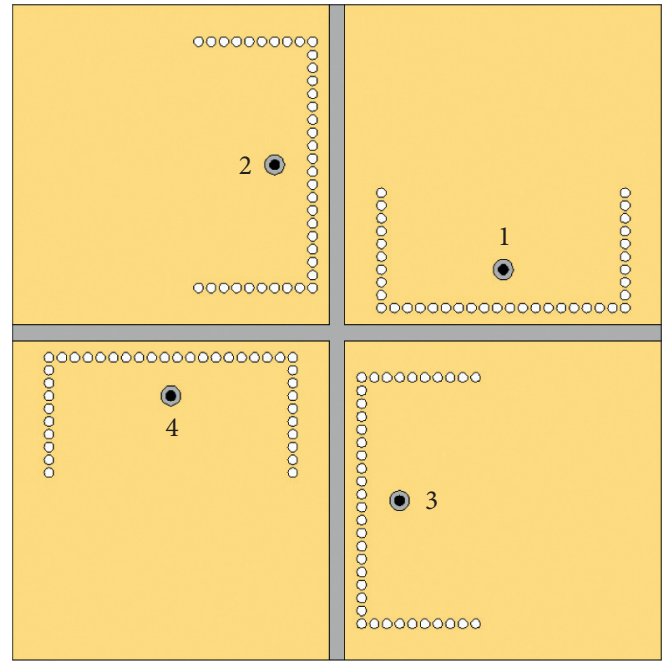

(b)

Figure 7: Geometry of the MIMO antenna with SNLs. (a) Top view and (b) bottom view.

where $F_{i}(\theta, \varphi)$ is a complex vector demonstrating the field radiating from the $i$ th element. The symbols "." and "*" indicate the Hermitian product and the complex conjugate, respectively. In this paper, the ECC is computed using both methods. For brevity, only $\rho_{12}$ and $\rho_{14}$ are presented here. As shown in Figures 14 and 15, the computed ECC is less than 0.005 across the two operating bands, which satisfies the requirement ECC < 0.5 and indicates good system performance. The $3 \mathrm{D}$ farfield method for finding ECC gives more accurate results [17].

DG determines the enhancement achieved by using multiple antenna systems over using single antenna systems. The relationship between ECC and DG can be described as [12]

$$
\mathrm{DG}=10 \sqrt{1-\left|0.99 \rho_{i j}\right|^{2}}
$$

It can be noted from (2) that a low ECC leads to ensure high DG. In an ideal case $\left(\rho_{i j}=0\right)$, the DG is equal to $10 \mathrm{~dB}$. As shown in Figure 16, the computed DG for the highest ECC values in the lower and upper operating bands is greater than $9.99 \mathrm{~dB}$ which guarantees good diversity performance.

TARC is the ratio of the square root of total reflected power divided by the square root of the total incident power. It can be computed using the following relation [18]:

$$
\Gamma_{a}^{t}=\frac{\sqrt{\sum_{i=1}^{N}\left|b_{i}\right|^{2}}}{\sqrt{\sum_{i=1}^{N}\left|a_{i}\right|^{2}}},
$$




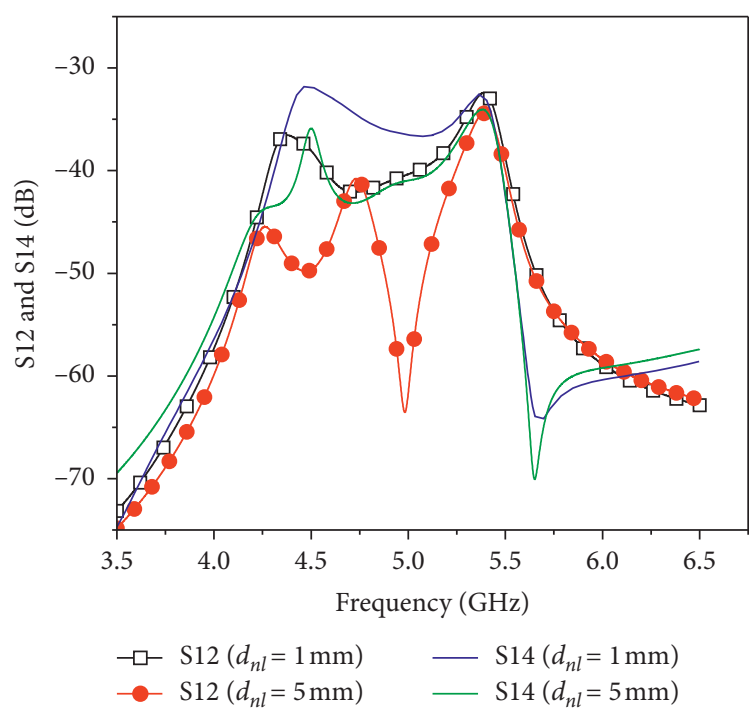

FIGURE 8: Simulated S12 and S14 of the MIMO antenna with variations in $d_{n l}$.

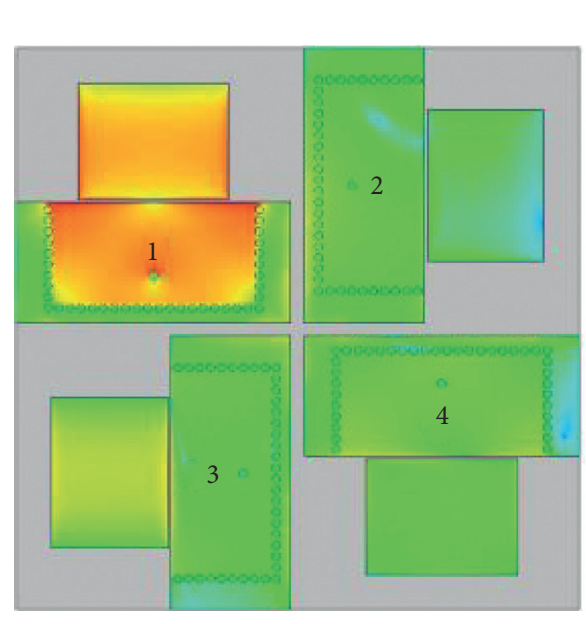

(a)

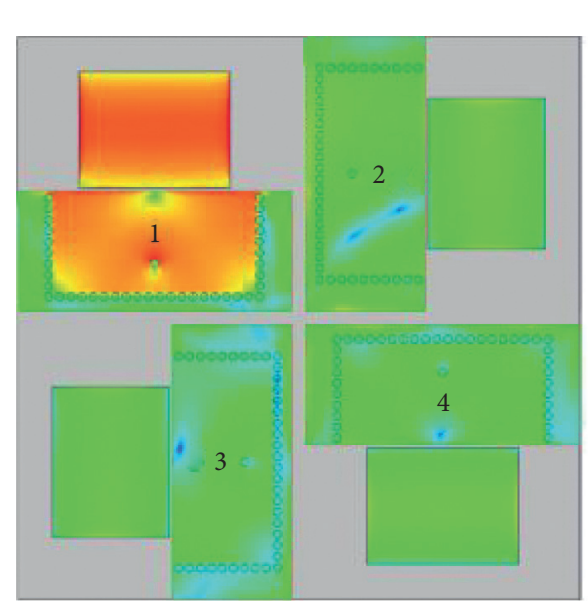

(c)
Jsurf [A_per_m]

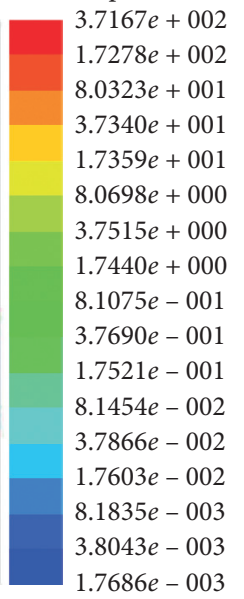

Jsurf [A_per_m]

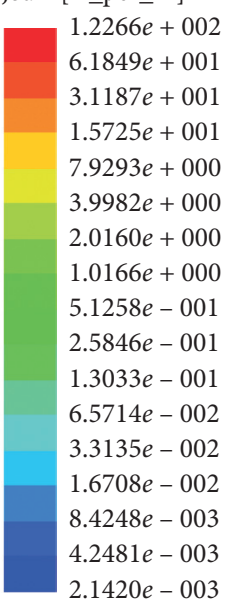

$2.1420 e-003$

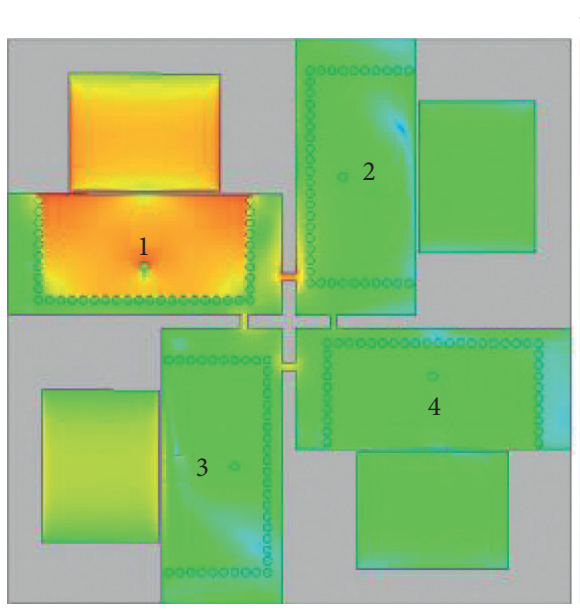

(b)

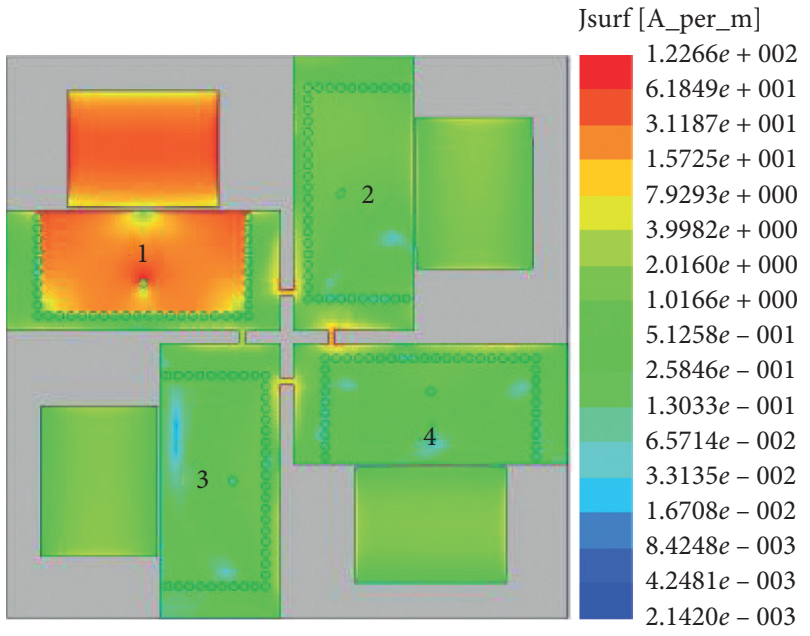

(d)
Jsurf [A_per_m]

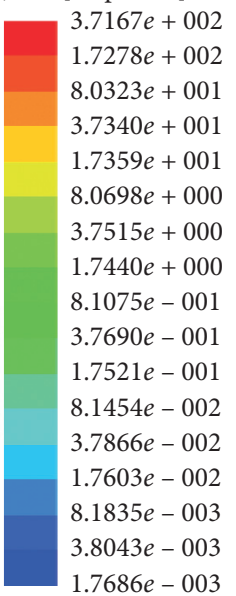

$1.7686 e-003$

Jsurf [A_per_m]

FIGURE 9: Current distribution when element 1 is excited and other elements are terminated to match loads. (a) 4.46 GHz, without SNLs. (b) $4.46 \mathrm{GHz}$, with SNLs. (c) $5.38 \mathrm{GHz}$, without SNLs. (d) $5.38 \mathrm{GHz}$, with SNLs. 


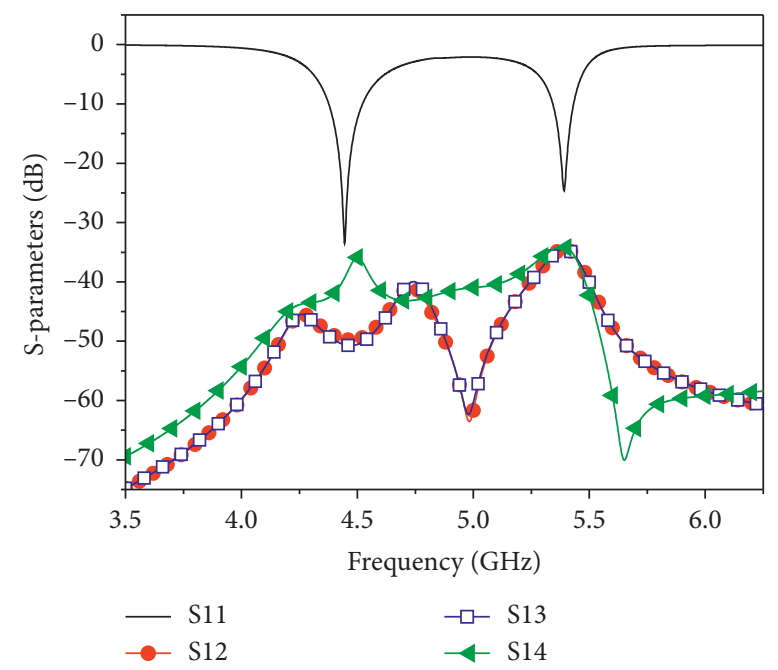

Figure 10: Simulated S-parameters of MIMO antenna with SNLs.

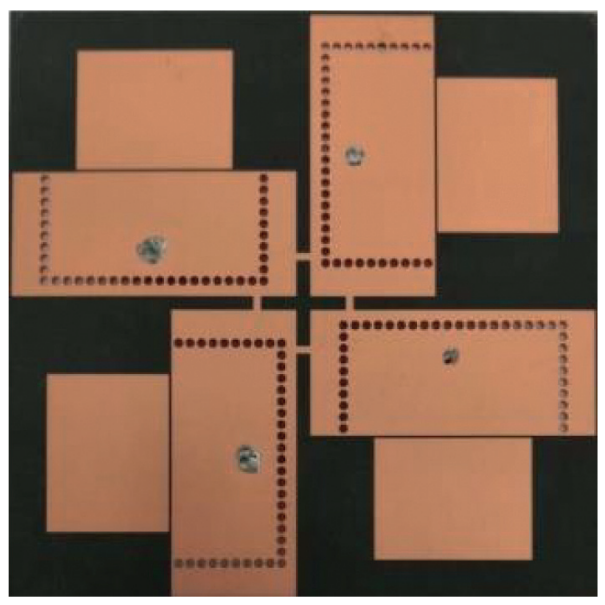

(a)

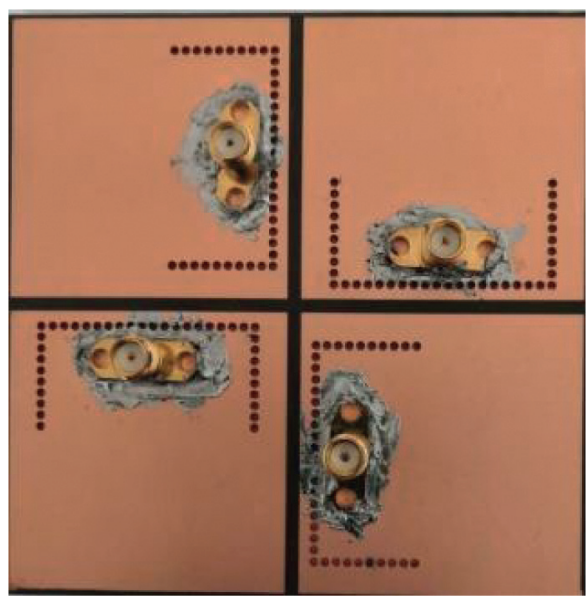

(b)

FIgURE 11: Photograph of the fabricated MIMO antenna with SNLs. (a) Top view and (b) bottom view.

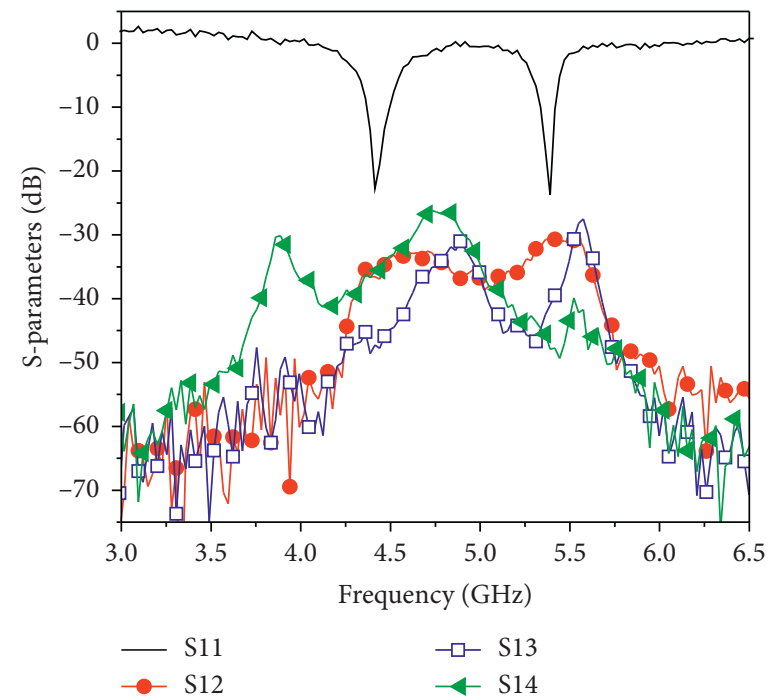

FIGURE 12: Measured S-parameters of the MIMO antenna with SNLs. 


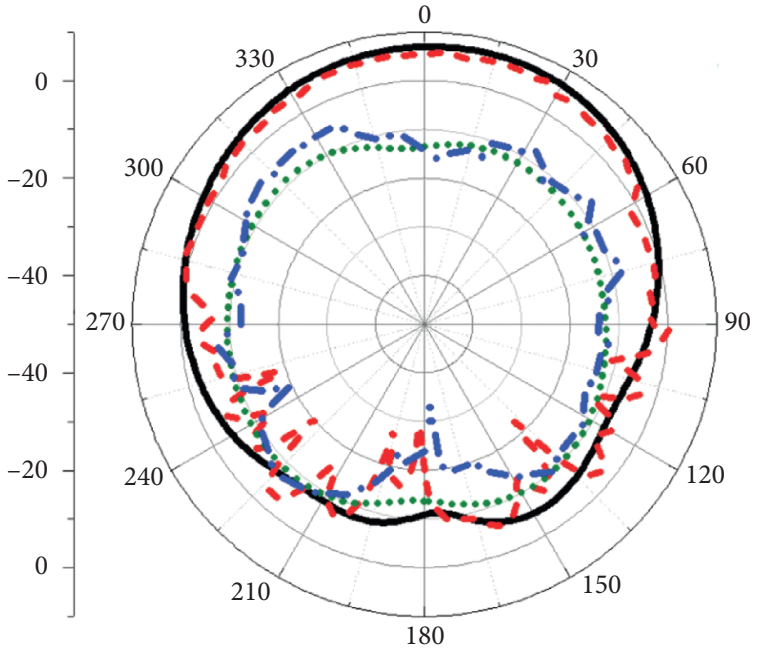

- Sim Co-P
-- Mea Co-P

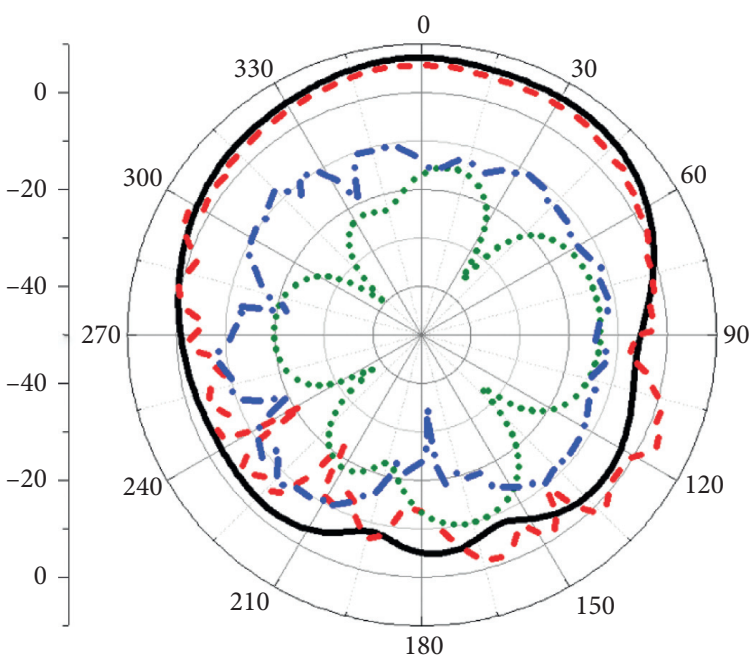

- Sim Co-P
-- Mea Co-P

...... Sim X-P

...- Mea X-P

(c)

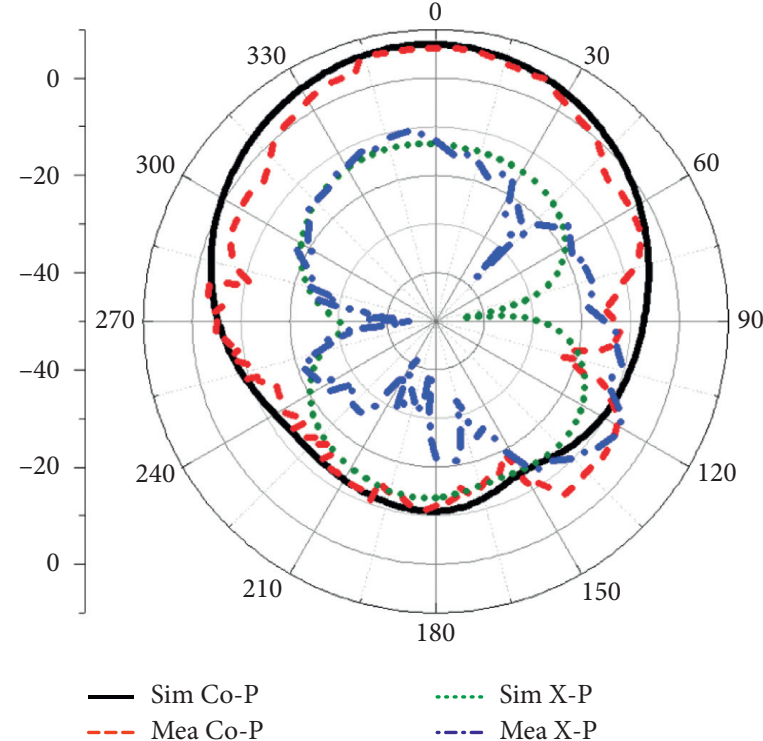

(b)

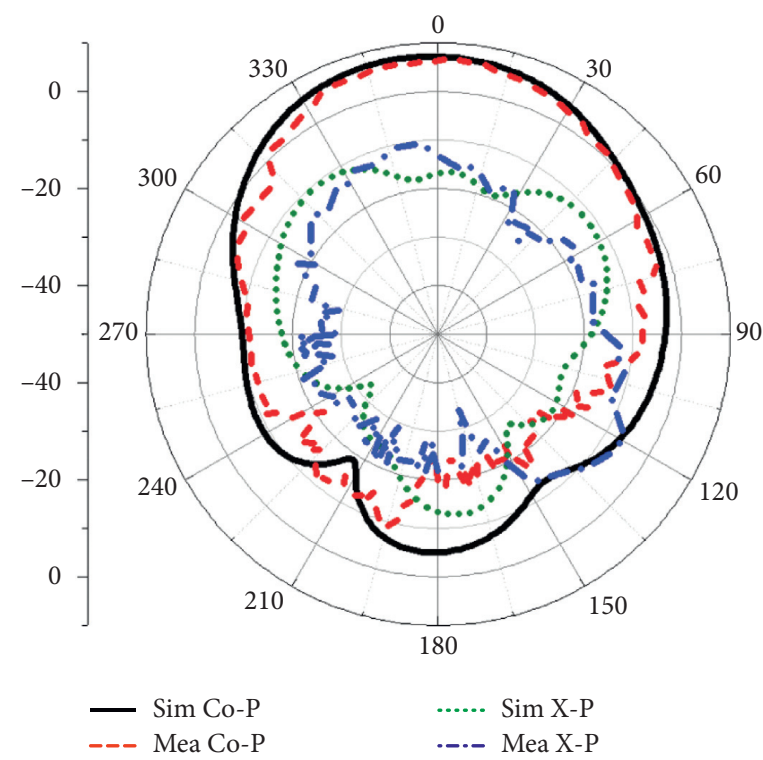

(d)

FIGURE 13: Simulated and measured radiation patterns of the proposed MIMO antenna with SNLs. (a) 4.46 GHz, E-plane ( $x z$-plane). (b) $4.46 \mathrm{GHz}, \mathrm{H}$-plane ( $y z$-plane). (c) $5.38 \mathrm{GHz}$, E-plane ( $x z$-plane). (d) $5.38 \mathrm{GHz}, \mathrm{H}$-plane (yz-plane).

where $b_{i}$ and $a_{i}$ represent reflected and incident signals, respectively, and $N$ is the number of antenna elements. The computed TARC is shown in Figure 17. As can be seen, the TARC is better than $-10 \mathrm{~dB}$ across the two operating bands.

MEG is defined as the ratio of the mean received power to the mean incident power of the antenna. If the antennas are $100 \%$ efficient, the maximum MEG is $-3 \mathrm{~dB}$ [19]. It can be computed using the following equation [20]:

$$
\text { MEG }=\frac{1}{2 \pi} \int_{0}^{2 \pi}\left[\frac{\Gamma}{1+\Gamma} \cdot G_{\theta}\left(\frac{\pi}{2}, \phi\right)+\frac{1}{1+\Gamma} \cdot G_{\phi}\left(\frac{\pi}{2}, \phi\right)\right] \mathrm{d} \phi,
$$

where $G_{\theta}$ and $G_{\varphi}$ are, respectively, $\theta$ and $\varphi$ polarized components of the antenna power gain pattern. $\Gamma$ is the cross-polarization discrimination of the incident field. The ratios of the MEG should satisfy the following condition in order to guarantee good channel characteristics [21]:

$$
\frac{\mathrm{MEG}_{i}}{\mathrm{MEG}_{j}} \cong 1
$$

In our design, average MEG of $-3.2 \mathrm{~dB}$ is achieved for all the four elements. The ratios of $M E G_{i} / M G_{j}$ are around 0.937 which satisfies condition (5). 


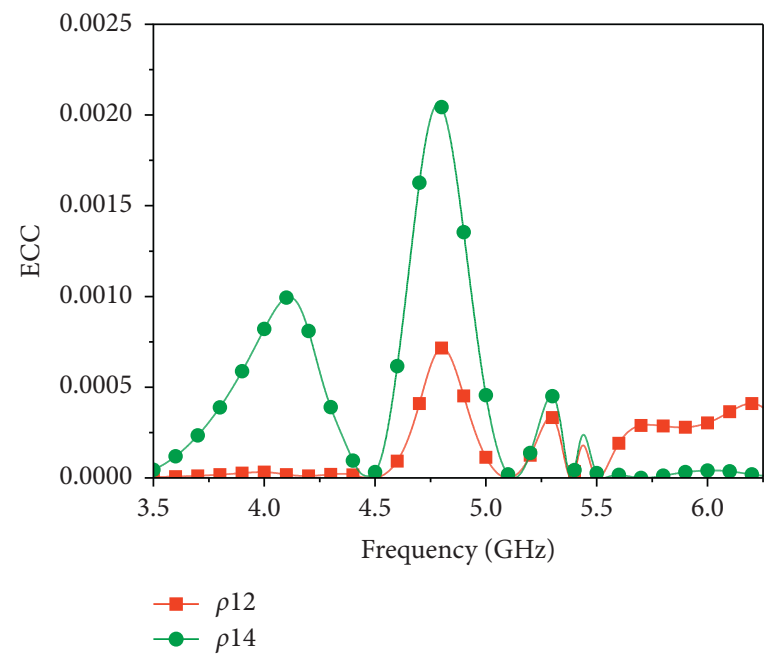

FIGURE 14: Computed ECC of the proposed MIMO antenna using S-parameters.

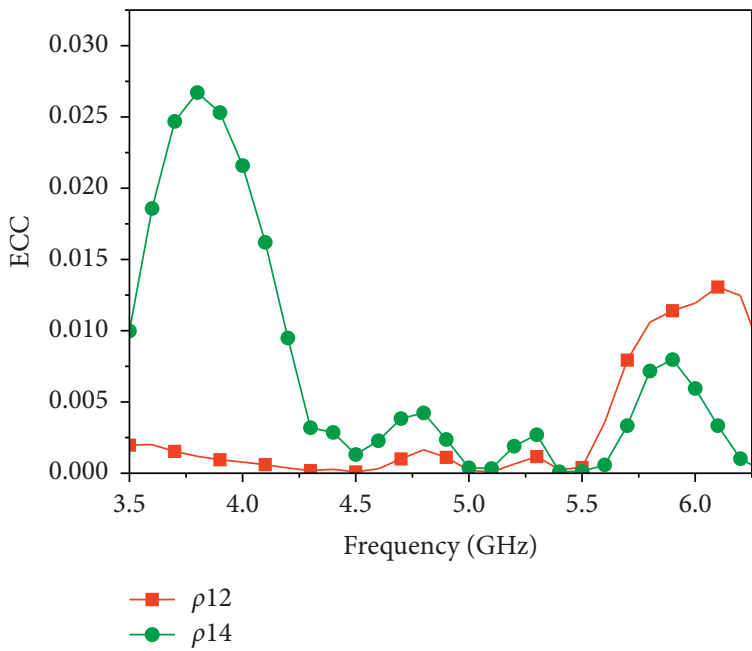

Figure 15: Computed ECC of the proposed MIMO antenna using three-dimensional far fields.

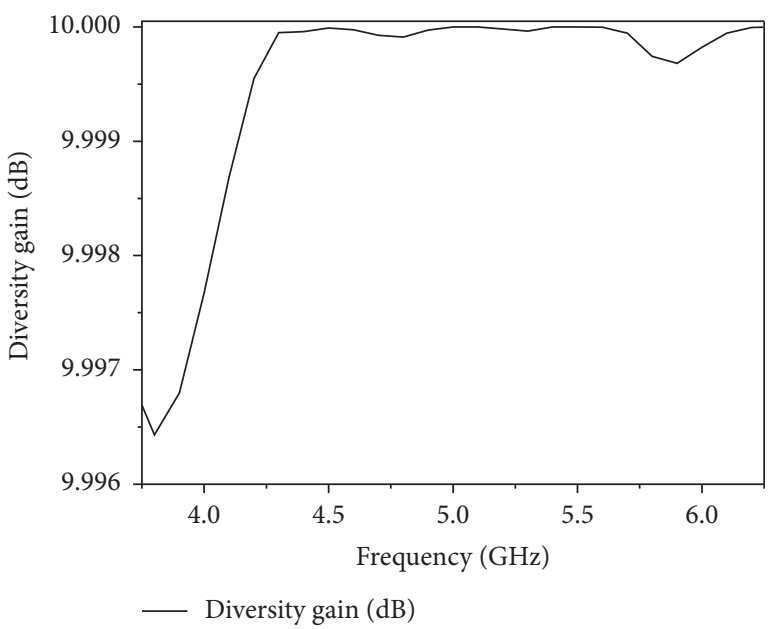

FIGURE 16: Computed diversity gain (DG) of the proposed MIMO antenna.
CCL caused by the correlation can be expressed as [22]

$$
C_{\text {loss }}=-\log _{2} \operatorname{det}\left(\Psi^{R}\right)
$$

where $\Psi^{R}$ is the correlation matrix. Figure 18 shows that the proposed MIMO antenna achieves lower than 0.2 CCL values (ideally, CCL should be $<0.4 \mathrm{bits} / \mathrm{s} / \mathrm{Hz}$ ) across the two operating bands. We can conclude that the diversity performances of the proposed MIMO antenna are suitable for a high-quality MIMO antenna system.

The performance of the proposed MIMO antenna is compared with that of the recent four-element MIMO antenna in Table 1 . Our $2 \times 2$ MIMO antenna has the highest isolation and gain. The proposed antenna also has the smallest edge-to-edge element spacing. The size of this work is smaller than that of the antenna in [14] and approximately equal to antenna in [15] and larger than that of the antennas in [11-13]. Our design has the smallest envelope correlation coefficient which guarantees good diversity performance. In this design, we used the coaxial probes to excite the dual- 


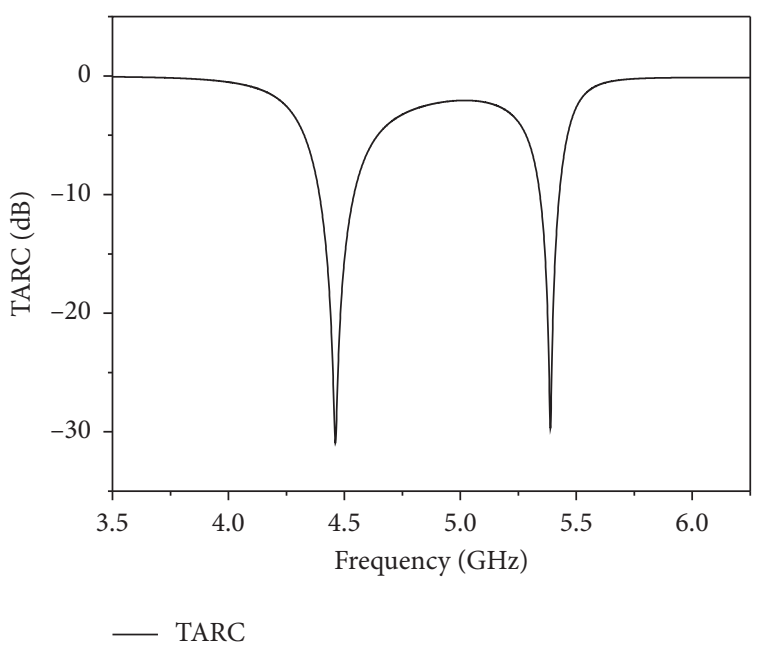

FigURE 17: Computed total active reflection coefficient (TARC) of the proposed MIMO antenna.

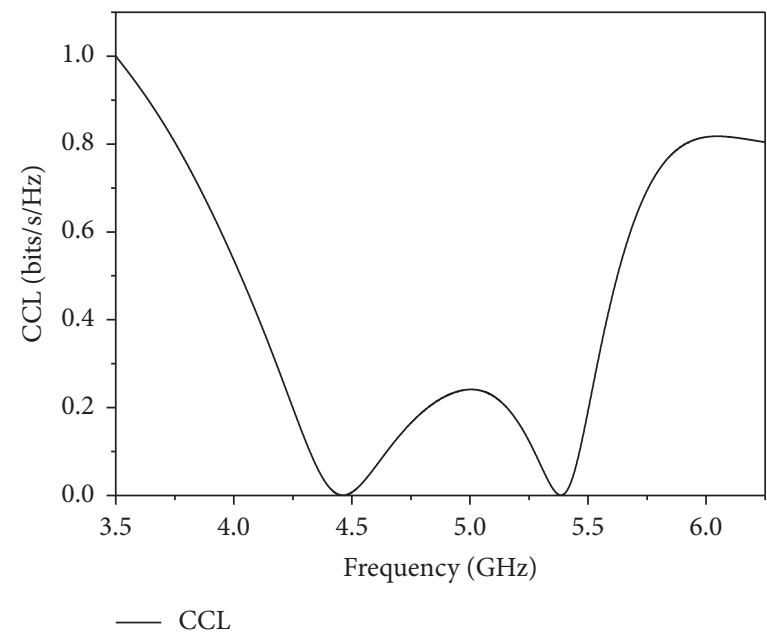

FIGURE 18: Computed channel capacity loss (CCL) of the proposed MIMO antenna.

TABle 1: Comparison study.

\begin{tabular}{|c|c|c|c|c|c|c|c|}
\hline Reference & $\begin{array}{c}\text { Center frequency } \\
(\mathrm{GHz})\end{array}$ & $\begin{array}{l}\text { Edge-to- } \\
\text { edge }\left(\lambda_{0}\right)\end{array}$ & $\begin{array}{l}\text { Gain } \\
(\mathrm{dBi}) \\
\end{array}$ & $\begin{array}{c}\text { Minimum } \\
\text { isolation }(\mathrm{dB})\end{array}$ & Total size & $\begin{array}{c}\text { Diversity } \\
\text { performance ECC }\end{array}$ & Excitation \\
\hline [11] & $2.45,5.50$ & 0.30 & NA & 17.5 & $\begin{array}{c}0.9 \lambda_{0} \times 0.9 \\
\lambda_{0} \times 0.015 \lambda_{0}\end{array}$ & 0.057 & Coaxial probe \\
\hline [12] & $3.50,5.70$ & 0.038 & $2.7,2.8$ & 18.4 & $\begin{array}{c}0.7 \lambda_{0} \times 0.7 \\
\lambda_{0} \times 0.075 \lambda_{0}\end{array}$ & 0.08 & Microstrip line \\
\hline [13] & $2.93,5.68$ & 0.226 & $4.0,4.0$ & 14 & $\begin{array}{c}0.8 \lambda_{0} \times 0.8 \\
\lambda_{0} \times 0.031 \quad \lambda_{0}\end{array}$ & 0.05 & Microstrip line \\
\hline [14] & $3.27,5.40$ & 0.447 & $4.8,5.3$ & 20 & $\begin{array}{c}1.6 \lambda_{0} \times 1.6 \\
\lambda_{0} \times 0.236 \lambda_{0}\end{array}$ & 0.06 & $\begin{array}{l}\text { Aperture } \\
\text { coupled }\end{array}$ \\
\hline$[15]$ & $2.54,5.26$ & 0.295 & $3.9,4.1$ & 21 & $\begin{array}{c}1.3 \lambda_{0} \times 1.3 \\
\lambda_{0} \times 0.027 \lambda_{0}\end{array}$ & 0.07 & Microstrip line \\
\hline This work & $4.43,5.39$ & 0.036 & $6,6.4$ & 35 & $\begin{array}{c}1.4 \lambda_{0} \times 1.4 \\
\lambda_{0} \times 0.028 \lambda_{0}\end{array}$ & 0.005 & Coaxial probe \\
\hline
\end{tabular}

$\lambda_{0}$ is the free-space wavelength at the upper-frequency band. NA = not available.

band antenna elements. In summary, our proposed $2 \times 2$ MIMO antenna has the highest isolation, highest gain, smallest edge-to-edge element spacing, and lowest envelope correlation coefficient.

\section{Conclusion}

In this paper, a close-spaced dual-band $2 \times 2 \mathrm{MIMO}$ antenna is proposed and fabricated. Good isolation was achieved by 
exploiting spatial and polarization diversities. An isolation enhancement of around $10 \mathrm{~dB}$ was achieved by inserting SNLs at the center of the MIMO antenna between the radiators of the antenna elements without increasing the size of the original MIMO antenna. With only $0.036 \lambda_{0}$ edge-toedge distance between elements, the measured isolation in both bands was higher than $35 \mathrm{~dB}$. Moreover, the diversity performances of the proposed MIMO antenna are suitable for a high-quality MIMO antenna system.

\section{Data Availability}

All simulated and measured data used to support the findings of this study are included within the article.

\section{Conflicts of Interest}

The authors declare that they have no conflicts of interest.

\section{Acknowledgments}

This work was supported by the National Natural Science Foundation of China under Grant 61771300.

\section{References}

[1] M. A. Jensen and J. W. Wallace, "A review of antennas and propagation for MIMO wireless communications," IEEE Transactions on Antennas and Propagation, vol. 52, no. 11, pp. 2810-2824, 2004.

[2] H. S. Singh, G. K. Pandey, P. K. Bharti, and M. K. Meshram, "A compact dual band MIMO/diversity antenna for WLAN applications,"in Proceedings of the SCES-2013, pp. 4, Allahabad,India, April 2013.

[3] T.-C. Hong and S.-W. Su, "Integrated, dual-band three-dipole-antenna system for single-radio, access-point applications," Microwave and Optical Technology Letters, vol. 53, no. 3, pp. 688-692, 2011.

[4] S. Yan, P. J. Soh, and G. A. E. Vandenbosch, "Dual-band textile MIMO antenna based on substrate-integrated waveguide (SIW) technology," IEEE Transactions on Antennas and Propagation, vol. 63, no. 11, pp. 4640-4647, 2015.

[5] M. S. Sharawi, A. B. Numan, M. U. Khan, and D. N. Aloi, "A dual-element dual-band MIMO antenna system with enhanced isolation for mobile terminals," IEEE Antennas and Wireless Propagation Letters, vol. 11, pp. 1006-1009, 2012.

[6] D.-G. Yang, D. O. Kim, and C.-Y. Kim, "Design of dual-band MIMO monopole antenna with high isolation using slotted CSRR for WLAN," Microwave and Optical Technology Letters, vol. 56, no. 10, pp. 2252-2257, 2014.

[7] X. Tan, W. Wang, Y. Wu, Y. Liu, and A. A. Kishk, "Enhancing isolation in dual-band meander-line multiple antenna by employing split EBG structure," IEEE Transactions on Antennas and Propagation, vol. 67, no. 4, pp. 2769-2774, 2019.

[8] Y. Yu, L. Yi, X. Liu, and Z. Gu, "Compact dual-frequency microstrip antenna array with increased isolation using neutralization lines," Progress In Electromagnetics Research Letters, vol. 56, pp. 95-100, 2015.

[9] L. Cui, J. Guo, Y. Liu, and C.-Y.-D. Sim, "An 8-element dualband MIMO antenna with decoupling stub for 5G smartphone applications," IEEE Antennas and Wireless Propagation Letters, vol. 18, no. 10, pp. 2095-2099, 2019.
[10] K.-L. Wong, P.-W. Lin, and H.-J. Hsu, "Decoupled WWAN/ LTE antennas with an isolation ring strip embedded therebetween for smartphone application," Microwave and Optical Technology Letters, vol. 55, no. 7, pp. 1470-1476, 2013.

[11] W.-J. Liao, C.-Y. Hsieh, B.-Y. Dai, and B.-R. Hsiao, "InvertedF/slot integrated dual-band four-antenna system for WLAN access points," IEEE Antennas and Wireless Propagation Letters, vol. 14, pp. 847-850, 2015.

[12] A. Boukarkar, X. Q. Lin, Y. Jiang, L. Y. Nie, P. Mei, and Y. Q. Yu, "A miniaturized extremely close-spaced four-element dual-band MIMO antenna system with polarization and pattern diversity," IEEE Antennas and Wireless Propagation Letters, vol. 17, no. 1, pp. 134-137, 2018.

[13] D. Sarkar and K. V. Srivastava, "Compact four-element SRRloaded dual-band MIMO antenna for WLAN/WiMAX/WiFi/ 4G-LTE and 5G applications," Electronics Letters, vol. 53, no. 25 , pp. 1623-1624, 2017.

[14] A. Sharma, A. Sarkar, A. Biswas, and M. J. Akhtar, "Dual-band multiple-input multiple-output antenna based on half split cylindrical dielectric resonator," Journal of Electromagnetic Waves and Applications, vol. 32, no. 9, pp. 1152-1163, 2018.

[15] L. Malviya, R. K. Panigrahi, and M. V. Kartikeyan, "A $2 \times 2$ dual-band mimo antenna with polarization diversity for wireless applications," Progress In Electromagnetics Research C, vol. 61, pp. 91-103, 2016.

[16] T. Deckmyn, S. Agneessens, A. C. F. Reniers et al., "A novel $60 \mathrm{GHz}$ wideband coupled half-mode/quarter-mode substrate integrated waveguide antenna," IEEE Transactions on Antennas and Propagation, vol. 65, no. 12, pp. 6915-6926, 2017.

[17] M. S. Sharawi, "Current misuses and future prospects for printed multiple-input, multiple-output antenna systems wireless corner," IEEE Antennas and Propagation Magazine, vol. 59, no. 2, pp. 162-170, 2017.

[18] S. H. Chae, S.-k. Oh, and S.-O. Park, "Analysis of mutual coupling, correlations, and TARC in WiBro MIMO array antenna," IEEE Antennas and Wireless Propagation Letters, vol. 6, pp. 122-125, 2007.

[19] S. C. K. Ko and R. D. Murch, "Compact integrated diversity antenna for wireless communications," IEEE Transactions on Antennas and Propagation, vol. 49, no. 6, pp. 954-960, 2001.

[20] Y. Ding, Z. Du, K. Gong, and Z. Feng, "A novel dual-band printed diversity antenna mobile terminals," IEEE Transactions on Antennas and Propagation, vol. 55, no. 7, pp. 2088-2096, 2007.

[21] M. Karaboikis, V. Papamichael, G. Tsachtsiris, C. Soras, and V. Makios, "Integrating compact printed antennas onto small diversity/MIMO terminals," IEEE Transactions on Antennas and Propagation, vol. 56, no. 7, pp. 2067-2078, 2008.

[22] X. Zhou, X. L. Quan, and R. L. Li, "A dual-broadband MIMO antenna system for GSM/UMTS/LTE and WLAN handsets," IEEE Antennas and Wireless Propagation Letters, vol. 11, pp. 551-554, 2012. 